\title{
Some properties of the sequence space
}

\section{$\widehat{B V} V_{\theta}(M, p, q, s)$}

Mahmut Işik', Yavuz Altin²* and Mikail Et²

\section{"Correspondence:}

yaltin23@yahoo.com

2 Department of Mathematics, Firat

University, Elazığ, 23119, Turkey

Full list of author information is

available at the end of the article

\begin{abstract}
In this paper we define the sequence space $\widehat{B V}_{\theta}(M, p, q, s)$ on a seminormed complex linear space by using an Orlicz function. We give various properties and some inclusion relations on this space.

MSC: 40A05; 40C05; 40D05
\end{abstract}

Keywords: Orlicz function; sequence spaces; seminorm

\section{Introduction}

Let $\ell_{\infty}$ and $c$ denote the Banach spaces of real bounded and convergent sequences $x=\left(x_{n}\right)$ normed by $\|x\|=\sup _{n}\left|x_{n}\right|$, respectively.

Let $\sigma$ be a one-to-one mapping of the set of positive integers into itself such that $\sigma^{k}(n)=$ $\sigma\left(\sigma^{k-1}(n)\right), k=1,2, \ldots$ A continuous linear functional $\varphi$ on $\ell_{\infty}$ is said to be an invariant mean or a $\sigma$-mean if and only if

(i) $\varphi(x) \geq 0$ when the sequence $x=\left(x_{n}\right)$ has $x_{n} \geq 0$ for all $n$,

(ii) $\varphi(e)=1$, where $e=(1,1,1, \ldots)$ and

(iii) $\varphi\left(\left\{x_{\sigma(n)}\right\}\right)=\varphi\left(\left\{x_{n}\right\}\right)$ for all $x \in \ell_{\infty}$.

If $\sigma$ is the translation mapping $n \rightarrow n+1$, a $\sigma$-mean is often called a Banach limit [1], and $V_{\sigma}$, the set of $\sigma$-convergent sequences, that is, the set of bounded sequences all of whose invariant means are equal, is the set $\hat{f}$ of almost convergent sequences [2].

If $x=\left(x_{n}\right)$, set $T x=\left(T x_{n}\right)=\left(x_{\sigma(n)}\right)$. It can be shown (see Schaefer [3]) that

$$
V_{\sigma}=\left\{x=\left(x_{n}\right): \lim _{k} t_{k n}(x)=\text { Le uniformly in } n, L=\sigma-\lim x\right\},
$$

where

$$
t_{k n}(x)=\frac{1}{k+1} \sum_{j=0}^{k} T^{j} x_{n} .
$$

The special case of (1.1), in which $\sigma(n)=n+1$, was given by Lorentz [2].

Subsequently invariant means were studied by Ahmad and Mursaleen [4], Mursaleen [5], Raimi [6] and many others.

\section{Springer}

(c) 2013 Issik et al.; licensee Springer. This is an Open Access article distributed under the terms of the Creative Commons Attribution License (http://creativecommons.org/licenses/by/2.0), which permits unrestricted use, distribution, and reproduction in any medium, provided the original work is properly cited. 
We may remark here that the concept $\hat{B V}$ of almost bounded variation was introduced and investigated by Nanda and Nayak [7] as follows:

$$
\widehat{B V}=\left\{x: \sum_{m}\left|t_{m n}(x)\right| \text { converges uniformly in } n\right\},
$$

where

$$
t_{m n}(x)=\frac{1}{m(m+1)} \sum_{v=1}^{m} v\left(x_{n+v}-x_{n+v-1}\right)
$$

By a lacunary sequence $\theta=\left(k_{r}\right)_{r=0,1,2, \ldots}^{\infty}$, where $k_{0}=0$, we shall mean an increasing sequence of non-negative integers with $k_{r}-k_{r-1} \rightarrow \infty$ as $r \rightarrow \infty$. The intervals determined by $\theta$ will be denoted by $I_{r}=\left(k_{r-1}, k_{r}\right]$, and we let $h_{r}=k_{r}-k_{r-1}$. The ratio $\frac{k_{r}}{k_{r-1}}$ will usually be denoted by $q_{r}$ (see [8]).

Karakaya and Savaş [9] defined the sequence spaces $\widehat{B V_{\theta}}(p)$ and $\widehat{B V_{\theta}}(p)$ as follows:

$$
\begin{aligned}
& \widehat{B V_{\theta}}(p)=\left\{x: \sum_{r=1}^{\infty}\left|\varphi_{r n}(x)\right|^{p_{r}} \text { converges uniformly in } n\right\}, \\
& \widehat{B V_{\theta}}(p)=\left\{x: \sup _{n} \sum_{r=1}^{\infty}\left|\varphi_{r n}(x)\right|^{p_{r}}<\infty\right\},
\end{aligned}
$$

where

$$
\varphi_{r, n}(x)=\frac{1}{h_{r}+1} \sum_{j=k_{r-1}+1} x_{j+n}-\frac{1}{h_{r}} \sum_{j=k_{r-1}+1}^{k_{r}} x_{j+n}, \quad r>1 .
$$

Straightforward calculation shows that

$$
\varphi_{r, n}(x)=\frac{1}{h_{r}\left(h_{r}+1\right)} \sum_{u=1}^{h_{r}} u\left(x_{k_{r-1}+u+1+n}-x_{k_{r-1}+u+n}\right)
$$

and

$$
\varphi_{r-1, n}(x)=\frac{1}{h_{r}\left(h_{r}-1\right)} \sum_{u=1}^{h_{r}-1}\left(x_{k_{r-1}+u+1+n}-x_{k_{r-1}+u+n}\right) .
$$

Note that for any sequences $x, y$ and scalar $\lambda$, we have

$$
\varphi_{r, n}(x+y)=\varphi_{r, n}(x)+\varphi_{r, n}(y) \quad \text { and } \quad \varphi_{r, n}(\lambda x)=\lambda \varphi_{r, n}(x) .
$$

An Orlicz function is a function $M:[0, \infty) \rightarrow[0, \infty)$, which is continuous, nondecreasing and convex with $M(0)=0, M(x)>0$ for $x>0$ and $M(x) \rightarrow \infty$ as $x \rightarrow \infty$. (For details, see Krasnoselskii and Rutickii [10].)

It is well known that if $M$ is a convex function and $M(0)=0$, then $M(\lambda x) \leq \lambda M(x)$ for all $\lambda$ with $0<\lambda<1$. 
Lindenstrauss and Tzafriri [11] used the idea of Orlicz function to construct the sequence space

$$
\ell_{M}=\left\{x \in w: \sum_{k=1}^{\infty} M\left(\frac{\left|x_{k}\right|}{\rho}\right)<\infty \text { for some } \rho>0\right\} .
$$

The space $\ell_{M}$ is a Banach space with the norm

$$
\|x\|=\inf \left\{\rho>0: \sum_{k=1}^{\infty} M\left(\frac{\left|x_{k}\right|}{\rho}\right) \leq 1\right\},
$$

and this space is called an Orlicz sequence space. For $M(t)=t^{p}, 1 \leq p<\infty$, the space $\ell_{M}$ coincides with the classical sequence space $\ell_{p}$.

Definition 1.1 Any two Orlicz functions $M_{1}$ and $M_{2}$ are said to be equivalent if there are positive constants $\alpha$ and $\beta$, and $x_{0}$ such that $M_{1}(\alpha x) \leq M_{2}(x) \leq M_{1}(\beta x)$ for all $x$ with $0 \leq x \leq x_{0}$ (see Kamthan and Gupta [12]).

Later on, different types of sequence spaces were introduced by using an Orlicz function by Mursaleen et al. [13], Choudhary and Parashar [14], Tripathy and Mahanta [15] , Altinok et al. [16], Bhardwaj and Singh [17], Et et al. [18] and many others.

A sequence space $E$ is said to be solid (or normal) if $\left(\alpha_{k} x_{k}\right) \in E$ whenever $\left(x_{k}\right) \in E$ for all sequences $\left(\alpha_{k}\right)$ of scalars with $\left|\alpha_{k}\right| \leq 1$.

It is well known that a sequence space $E$ is normal implies that $E$ is monotone.

Definition 1.2 Let $q_{1}, q_{2}$ be seminorms on a vector space $X$. Then $q_{1}$ is said to be stronger than $q_{2}$ if whenever $\left(x_{n}\right)$ is a sequence such that $q_{1}\left(x_{n}\right) \rightarrow 0$, then also $q_{2}\left(x_{n}\right) \rightarrow 0$. If each is stronger than the others, $q_{1}$ and $q_{2}$ are said to be equivalent (one may refer to Wilansky [19]).

Lemma 1.3 Let $q_{1}$ and $q_{2}$ be seminorms on a linear space $X$. Then $q_{1}$ is stronger than $q_{2}$ if and only if there exists a constant $T$ such that $q_{2}(x) \leq T q_{1}(x)$ for all $x \in X$ (see, for instance, Wilansky [19]).

Let $p=\left(p_{r}\right)$ be a sequence of strictly positive real numbers, $X$ be a seminormed space over the field $\mathbb{C}$ of complex numbers with the seminorm $q, M$ be an Orlicz function and $s \geq 0$ be a fixed real number. Then we define the sequence space $\widehat{B V}(M, p, q, s)$ as follows:

$$
\begin{aligned}
\widehat{B V} V_{\theta}(M, p, q, s)= & \left\{x=\left(x_{k}\right) \in X: \sum_{r=1}^{\infty} r^{-s}\left[M\left(q\left(\frac{\varphi_{r n}(x)}{\rho}\right)\right)\right]^{p_{r}}<\infty\right. \\
& \text { for some } \rho>0 \text { uniformly in } n\} .
\end{aligned}
$$

It is clear that $q\left(\frac{\varphi_{r n}(x)}{\rho}\right)=\frac{q\left(\varphi_{r n}(x)\right)}{\rho}$ for any seminorm $q$ and any $\rho>0$.

We get the following sequence spaces from $B V_{\theta}(M, p, q, s)$ by choosing some of the special $p, M$ and $s$ : 
For $M(x)=x$ we get

$$
\widehat{B V_{\theta}}(p, q, s)=\left\{x=\left(x_{k}\right) \in X: \sum_{r=1}^{\infty} r^{-s}\left[\left(q\left(\varphi_{r n}(x)\right)\right)\right]^{p_{r}}<\infty \text { uniformly in } n\right\}
$$

for $p_{k}=1$, for all $r \in \mathbb{N}$, we get

$$
\begin{aligned}
& \widehat{B V_{\theta}}(M, q, s) \\
& \quad=\left\{x=\left(x_{k}\right) \in X: \sum_{r=1}^{\infty} r^{-s}\left[M\left(q\left(\frac{\varphi_{r n}(x)}{\rho}\right)\right)\right]<\infty \text { for some } \rho>0 \text { uniformly in } n\right\}
\end{aligned}
$$

for $s=0$ we get

$$
\begin{aligned}
& \widehat{B V_{\theta}}(M, p, q) \\
& \quad=\left\{x=\left(x_{k}\right) \in X: \sum_{r=1}^{\infty}\left[M\left(q\left(\frac{\varphi_{r n}(x)}{\rho}\right)\right)\right]^{p_{r}}<\infty \text { for some } \rho>0 \text { uniformly in } n\right\}
\end{aligned}
$$

for $M(x)=x$ and $s=0$ we get

$$
\widehat{B V_{\theta}}(p, q)=\left\{x=\left(x_{k}\right) \in X: \sum_{r=1}^{\infty}\left[\left(q\left(\varphi_{r n}(x)\right)\right)\right]^{p_{r}}<\infty \text { uniformly in } n\right\}
$$

for $p_{r}=1$, for all $r \in \mathbb{N}$, and $s=0$ we get

$$
\begin{aligned}
& \widehat{B V_{\theta}(M, q)} \\
& \quad=\left\{x=\left(x_{k}\right) \in X: \sum_{r=1}^{\infty}\left[M\left(q\left(\frac{\varphi_{r n}(x)}{\rho}\right)\right)\right]<\infty \text { for some } \rho>0 \text { uniformly in } n\right\}
\end{aligned}
$$

for $M(x)=x, p_{r}=1$, for all $r \in \mathbb{N}$, and $s=0$ we have

$$
B V_{\theta}(q)=\left\{x=\left(x_{k}\right) \in X: \sum_{r=1}^{\infty} q\left(\varphi_{r n}(x)\right)<\infty, \text { uniformly in } n\right\} .
$$

The following inequalities will be used throughout the paper. Let $p=\left(p_{r}\right)$ be a bounded sequence of strictly positive real numbers with $0<p_{r} \leq \sup p_{r}=H, D=\max \left(1,2^{H-1}\right)$, then

$$
\left|a_{r}+b_{r}\right|^{p_{r}} \leq D\left\{\left|a_{r}\right|^{p_{r}}+\left|b_{r}\right|^{p_{r}}\right\}
$$

where $a_{r}, b_{r} \in \mathbb{C}$.

\section{Main results}

In this section we prove the general results of this paper on the sequence space $\widehat{B V} V_{\theta}(M, p$, $q, s)$, those characterize the structure of this space.

Theorem 2.1 The sequence space $\widehat{B V} V_{\theta}(M, p, q, s)$ is a linear space over the field $\mathbb{C}$ of complex numbers. 
Proof Omitted.

Theorem 2.2 For any Orlicz function $M$ and a bounded sequence $p=\left(p_{r}\right)$ of strictly positive real numbers, $\widehat{B V}_{\theta}(M, p, q, s)$ is a paranormed space (not necessarily totally paranormed), paranormed by

$$
\begin{gathered}
g(x)=\inf \left\{\rho^{p_{r} / H}:\left(\sum_{r=1}^{\infty} r^{-s}\left[M\left(q\left(\frac{\varphi_{r n}(x)}{\rho}\right)\right)\right]^{p_{k}}\right)^{\frac{1}{H}} \leq 1,\right. \\
r=1,2,3, \ldots, n=1,2,3, \ldots\},
\end{gathered}
$$

where $H=\max \left(1, \sup p_{r}\right)$.

Proof Clearly $g(x)=g(-x)$. By using Theorem 2.1 and then using Minkowski's inequality, we get $g(x+y) \leq g(x)+g(y)$.

Since $q(\bar{\theta})=0$ and $M(0)=0$, we get $\inf \left\{\rho^{p_{r} / H}\right\}=0$ for $x=\Theta$, where $\bar{\Theta}$ is the zero sequence of $X$.

Finally, we prove that scalar multiplication is continuous. Let $\lambda$ be any numbers. By definition,

$$
\begin{gathered}
g(\lambda x)=\inf \left\{\rho^{p_{r} / H}:\left(\sum_{r} r^{-s}\left[M\left(q\left(\frac{\lambda \varphi_{r n}(x)}{\rho}\right)\right)\right]^{p_{r}}\right)^{\frac{1}{H}} \leq 1,\right. \\
r=1,2,3, \ldots, n=1,2,3, \ldots\} .
\end{gathered}
$$

Then

$$
\begin{gathered}
g(\lambda x)=\inf \left\{(\lambda r)^{p_{r} / H}:\left(\sum_{r=1}^{\infty} r^{-s}\left[M\left(q\left(\frac{\varphi_{r n}(x)}{r}\right)\right)\right]^{p_{r}}\right)^{\frac{1}{H}} \leq 1,\right. \\
r=1,2,3, \ldots, n=1,2,3, \ldots\},
\end{gathered}
$$

where $r=\frac{\rho}{|\lambda|}$. Since $|\lambda|^{p_{r}} \leq \max \left(1,|\lambda|^{H}\right)$, it follows that $|\lambda|^{p_{r} / H} \leq\left(\max \left(1,|\lambda|^{H}\right)\right)^{\frac{1}{H}}$.

Hence

$$
\begin{gathered}
g(\lambda x)=\left(\max \left(1,|\lambda|^{H}\right)\right)^{\frac{1}{H}} \inf \left\{r^{p_{r} / H}:\left(\sum_{r=1}^{\infty} r^{-s}\left[M\left(q\left(\frac{\varphi_{r n}(x)}{r}\right)\right)\right]^{p_{r}}\right)^{\frac{1}{H}} \leq 1,\right. \\
r=1,2,3, \ldots, n=1,2,3, \ldots\},
\end{gathered}
$$

which converges to zero as $g(x)$ converges to zero in $\widehat{B V}_{\theta}(M, p, q, s)$. Now suppose that $\lambda_{n} \rightarrow 0$ and $x$ is in $B V_{\sigma}(M, p, q, s)$. For arbitrary $\varepsilon>0$, let $N$ be a positive integer such that

$$
\sum_{r=N+1}^{\infty} r^{-s}\left[M\left(q\left(\frac{\varphi_{r n}(x)}{\rho}\right)\right)\right]^{p_{r}}<\frac{\varepsilon}{2}
$$


for some $\rho>0$, all $n$. This implies that

$$
\left(\sum_{r=N+1}^{\infty} r^{-s}\left[M\left(q\left(\frac{\varphi_{r n}(x)}{\rho}\right)\right)\right]^{p_{r}}\right)^{\frac{1}{H}} \leq \frac{\varepsilon}{2}
$$

for some $\rho>0, r>N$ and all $n$.

Let $0<|\lambda|<1$, using convexity of $M$ and all $n$, we get

$$
\sum_{r=N+1}^{\infty} r^{-s}\left[M\left(q\left(\frac{\lambda \varphi_{r n}(x)}{\rho}\right)\right)\right]^{p_{r}}<\sum_{r=N+1}^{\infty} r^{-s}\left[|\lambda| M\left(q\left(\frac{\varphi_{r n}(x)}{\rho}\right)\right)\right]^{p_{r}}<\left(\frac{\varepsilon}{2}\right)^{H} .
$$

Since $M$ is continuous everywhere in $[0, \infty)$, then

$$
f(t)=\sum_{r=1}^{N} r^{-s}\left[M\left(q\left(\frac{t \varphi_{r n}(x)}{\rho}\right)\right)\right]
$$

is continuous at 0 . So there is $1>\delta>0$ such that $|f(t)|<\frac{\varepsilon}{2}$ for $0<t<\delta$. Let $K$ be such that $\left|\lambda_{i}\right|<\delta$ for $i>K$, then for $i>K$, all $n$,

$$
\left(\sum_{r=1}^{N} r^{-s}\left[M\left(q\left(\frac{\lambda_{i} \varphi_{r n}(x)}{\rho}\right)\right)\right]^{p_{r}}\right)^{\frac{1}{H}}<\frac{\varepsilon}{2} .
$$

Thus

$$
\left(\sum_{r=1}^{\infty} r^{-s}\left[M\left(q\left(\frac{\lambda_{i} \varphi_{r n}(x)}{\rho}\right)\right)\right]^{p_{r}}\right)^{\frac{1}{H}}<\varepsilon
$$

for $i>K$ and $n$, so that $g(\lambda x) \rightarrow 0(\lambda \rightarrow 0)$.

Theorem 2.3 Let $M, M_{1}, M_{2}$ be Orlicz functions $q, q_{1}, q_{2}$ seminorms and $s, s_{1}, s_{2} \geq 0$. Then

(i) $\hat{B V} V_{\theta}\left(M_{1}, p, q, s\right) \cap \hat{B V}_{\theta}\left(M_{2}, p, q, s\right) \subseteq \hat{B V} V_{\theta}\left(M_{1}+M_{2}, p, q, s\right)$,

(ii) If $s_{1} \leq s_{2}$ then $\hat{B V} V_{\theta}\left(M, p, q, s_{1}\right) \subseteq B V_{\theta}\left(M, p, q, s_{2}\right)$,

(iii) $\widehat{B V} V_{\theta}\left(M, p, q_{1}, s\right) \cap \widehat{B V}\left(M, p, q_{2}, s\right) \subseteq \widehat{B} V_{\theta}\left(M, p, q_{1}+q_{2}, s\right)$,

(iv) If $q_{1}$ is stronger than $q_{2}$, then $\widehat{B V}_{\theta}\left(M, p, q_{1}, s\right) \subseteq \widehat{B V} V_{\theta}\left(M, p, q_{2}, s\right)$.

Proof Omitted

Corollary 2.4 Let $M$ be an Orlicz function, then we have

(i) If $q_{1} \cong\left(\right.$ equivalent to) $q_{2}$, then $\widehat{B V} V_{\theta}\left(M, p, q_{1}, s\right)=\widehat{B V} V_{\theta}\left(M, p, q_{2}, s\right)$,

(ii) $\widehat{B V} V_{\theta}(M, p, q) \subseteq \widehat{B V} V_{\theta}(M, p, q, s)$,

(iii) $\widehat{B V} V_{\theta}(M, q) \subseteq \widehat{B V} V_{\theta}(M, q, s)$.

Theorem 2.5 Suppose that $0<m_{k} \leq t_{k}<\infty$ for each $k \in \mathbb{N}$. Then $\hat{B V}_{\theta}(M, m, q) \subseteq$ $\widehat{B V}_{\theta}(M, t, q)$. 
Proof Let $x \in \hat{B V}(M, m, q)$. Then there exists some $\rho>0$ such that

$$
\sum_{r=1}^{\infty}\left[M\left(q\left(\frac{\varphi_{r n}(x)}{\rho}\right)\right)\right]^{m_{k}}<\infty \quad \text { uniformly in } n .
$$

This implies that $M\left(q\left(\frac{\varphi_{r n}(x)}{\rho}\right)\right) \leq 1$ for sufficiently large values of $k$, say $k \geq k_{0}$ for some fixed $k_{0} \in \mathbb{N}$. Since $m_{k} \leq t_{k}$, for each $k \in \mathbb{N}$ we get

$$
\left[M\left(q\left(\frac{\varphi_{r n}(x)}{\rho}\right)\right)\right]^{t_{k}} \leq\left[M\left(q\left(\frac{\varphi_{r n}(x)}{\rho}\right)\right)\right]^{m_{k}}
$$

for all $k \geq k_{0}$, and therefore

$$
\sum_{r=1}^{\infty}\left[M\left(q\left(\frac{\varphi_{r n}(x)}{\rho}\right)\right)\right]^{t_{r}} \leq \sum_{r=1}^{\infty}\left[M\left(q\left(\frac{\varphi_{m}(x)}{\rho}\right)\right)\right]^{m_{k}} .
$$

Hence we have

$$
\sum_{r=1}^{\infty}\left[M\left(q\left(\frac{\varphi_{r n}(x)}{\rho}\right)\right)\right]^{t_{r}}<\infty,
$$

so $x \in \hat{B V}{ }_{\theta}(M, t, q)$. This completes the proof.

The following result is a consequence of the above result.

\section{Corollary 2.6}

(i) If $0<p_{r} \leq 1$ for each $r$, then $\widehat{B V} V_{\theta}(M, p, q) \subseteq \widehat{B} V_{\theta}(M, q)$,

(ii) If $p_{r} \geq 1$ for all $r$, then $\widehat{B V}_{\theta}(M, q) \subseteq \widehat{B V} V_{\theta}(M, p, q)$.

Theorem 2.7 Let $M_{1}$ and $M_{2}$ be any two of Orlicz functions. If $M_{1}$ and $M_{2}$ are equivalent, then $\widehat{B V}_{\theta}\left(M_{1}, p, q, s\right)=\widehat{B V}_{\theta}\left(M_{2}, p, q, s\right)$.

Proof Proof follows from Definition 1.1.

Theorem 2.8 The sequence space $\widehat{B V}_{\theta}(M, p, q, s)$ is solid.

Proof Let $x \in \widehat{B V_{\theta}}(M, p, q, s)$, i.e.,

$$
\sum_{r=1}^{\infty} r^{-s}\left[M\left(q\left(\frac{\varphi_{r n}(x)}{\rho}\right)\right)\right]^{p_{r}}<\infty .
$$

Let $\left(\alpha_{r}\right)$ be sequence of scalars such that $\left|\alpha_{r}\right| \leq 1$ for all $r \in \mathbb{N}$. Then the result follows from the following inequality:

$$
\sum_{r=1}^{\infty} r^{-s}\left[M\left(q\left(\frac{\alpha_{r} \varphi_{r n}(x)}{\rho}\right)\right)\right]^{p_{r}} \leq \sum_{r=1}^{\infty} r^{-s}\left[M\left(q\left(\frac{\varphi_{r n}(x)}{\rho}\right)\right)\right]^{p_{r}} .
$$

Corollary 2.9 The sequence space $\widehat{B V}_{\theta}(M, p, q, s)$ is monotone. 


\section{Competing interests}

The authors declare that they have no competing interest.

\section{Authors' contributions}

$\mathrm{MI}, \mathrm{YA}$ and ME have contributed to all parts of the article. All authors read and approved the final manuscript.

\section{Author details}

${ }^{1}$ Department of Statistics, Firat University, Elazığ, 23119, Turkey. ${ }^{2}$ Department of Mathematics, Firat University, Elazığ, 23119, Turkey.

\section{Received: 10 April 2013 Accepted: 16 June 2013 Published: 2 July 2013}

\section{References}

1. Banach, S: Theorie des Operations Linearies, Subwncji Funduszu Narodowej, Warszawa (1932)

2. Lorentz, GG: A contribution the theory of divergent series. Acta Math. 80, 167-190 (1948)

3. Schaefer, P: Infinite matrices and invariant means. Proc. Am. Math. Soc. 36, 104-110 (1972)

4. Ahmad, ZU, Mursaleen, M: An application of Banach limits. Proc. Am. Math. Soc. 103, 244-246 (1988)

5. Mursaleen, M: Matrix transformations between some new sequence spaces. Houst. J. Math. 9, 505-509 (1983)

6. Raimi, RA: Invariant means and invariant matrix method of summability. Duke Math. J. 30, 81-94 (1963)

7. Nanda, S, Nayak, KC: Some new sequence spaces. Indian J. Pure Appl. Math. 9(8), 836-846 (1978)

8. Freedman, AR, Sember, JJ, Raphael, M: Some Cesàro-type summability spaces. Proc. Lond. Math. Soc. 37(3), 508-520 (1978)

9. Karakaya, V, Savaş, E: On almost p-bounded variation of lacunary sequences. Comput. Math. Appl. 61(6), 1502-1506 (2011)

10. Krasnoselskii, MA, Rutickii, YB: Convex Functions and Orlicz Spaces. Noordhoff, Groningen (1961)

11. Lindenstrauss, J, Tzafriri, L: On Orlicz sequence spaces. Isr. J. Math. 10, 379-390 (1971)

12. Kamthan, PK, Gupta, M: Sequence Spaces and Series. Lecture Notes in Pure and Applied Mathematics, vol. 65. Dekker, New York (1981)

13. Mursaleen, M, Khan, QA, Chishti, TA: Some new convergent sequences defined by Orlicz functions and statistical convergence. Ital. J. Pure Appl. Math. 9, 25-32 (2001)

14. Choudhary, B, Parashar, SD: A sequence space defined by Orlicz functions. J. Approx. Theory Appl. 18(4), 70-75 (2002)

15. Tripathy, BC, Mahanta, S: On a class of sequences related to the $\ell^{p}$ spaces defined by Orlicz function. Soochow J. Math. 29(4), 379-391 (2003)

16. Altinok, $H$, Altin, $Y$, Işik, $M$ : The sequence space $B V_{\sigma}(M, p, q, s)$ on seminormed spaces. Indian J. Pure Appl. Math. 39(1), 49-58 (2008)

17. Bhardwaj, VK, Singh, N: On some new spaces of lacunary strongly $\sigma$-convergent sequences defined by Orlicz functions. Indian J. Pure Appl. Math. 31(11), 1515-1526 (2000)

18. Et, M, Altin, Y, Choudhary, B, Tripathy, BC: On some classes of sequences defined by sequences of Orlicz functions. Math. Inequal. Appl. 9(2), 335-342 (2006)

19. Wilansky, A: Functional Analysis. Blaisdell Publishing Company, New York (1964)

doi:10.1186/1029-242X-2013-305

Cite this article as: Işik et al.: Some properties of the sequence space $\widehat{B V} V_{\theta}(M, p, q, s)$. Journal of Inequalities and Applications 2013 2013:305. 